\title{
PAGE USAGE IN A QUADTREE INDEX
}

\author{
MAMORU HOSHI* and PHILIPPE FLAJOLET** \\ Faculty of Engineering, \\ Chiba University, \\ I-33 Yayoi-Cho, Chiba \\ Japan 260 \\ Algorithms Project, \\ INRIA, Rocquencourt, \\ F-78153 Le Chesnay \\ France
}

\begin{abstract}
.
This paper provides a characterization of the storage needs of a quadtree when used as an index to access large volumes of 2-dimensional data. It is shown that the page occupancy for data in random order approaches $33 \%$. A precise mathematical analysis that involves a modicum of hypergeometric functions and dilogarithms, together with some computer algebra is presented.

A brief survey of the analysis of storage usage in tree structures is included. The $33 \%$ ratio for quadtrees is to be compared to the figures for binary search trees $(50 \%)$, tries $(69 \%)$, and quadtries $(46 \%)$.
\end{abstract}

Computing Reviews Classification: E.1, E.2, F.2.2, G.2.1.

\section{Introduction.}

The quadtree structure is a fundamental hierarchical representation of point data in higher dimensional spaces. It was invented by Finkel and Bentley in 1974 [7], and it constitutes a natural generalization of binary search trees to multidimensional data. Under one form or other, it has surfaced in many different fields, like data bases, geographical data processing, graphics and image processing. A comprehensive treatment of this area of algorithmic design is to be found in Samet's book [23].

We discuss here the (point) quadtrees, for data in 2-dimensional space. More precisely, we concentrate on quadtrees that depend on an integer parameter $b \geq 0$ representing a page capacity, sometimes also called a bucket capacity; small subfiles (i.e., with size $\leq b$ ) are represented sequentially into a page instead of being split recursively.

The paged quadtrees that we consider thus naturally occur if one is to maintain large collections of data on external storage using the quadtree principle. They can

* The research of this author was done while visiting INRIA, Rocquencourt, France under support from the Ministry of Education of Japanese Government.

** Work of this author was supported in part by the Basic Research Action of the E.C. under contract No. 3075 (Project ALCOM).

Received May 1991. Revised December 1991. 
also be useful even as direct (in core) data structures since they build a hierarchical cell decomposition: If $b$ is large enough, nearest neighbours of a point are very likely to be found in the same cell (page); in this way nearest neighbour queries can be answered by a simple local search which is fairly efficient and adaptive.

Our major results characterize the expected storage occupancy of quadtres. For data in random order, we establish that the filling ratio of pages is approximately $33 \%$, in the sense that the number of pages necessary to store a file of $n$ points with $b$ the page capacity is about $3 n / b$.

Our precise results are the following.

THEOREM 1. Given a page capacity $b \geq 1$, there exists a constant $\gamma_{b}$ such that the expected number of pages for a paged quadtree with page capacity $b$ built on $n$ random points satisfies

$$
P_{n}^{[b]}=\gamma_{b} \cdot n+O(\log n)
$$

where $\gamma_{b}$ is

$$
\gamma_{b}=9 \int_{0}^{1} \frac{(1-t)^{3}}{t(1+2 t)^{2}}\left[\int_{0}^{t} \frac{(1+2 v)}{(1-v)^{2}} E_{b}(v) d v\right] d t
$$

with

$$
E_{b}(z)=z^{b} \frac{1+b(1-z)+b(b+1)(1-z)^{2}}{(1-z)^{2}}
$$

Table 1. Numerical values of the constant $\gamma_{b}$ and of $b \gamma_{b}$ for various values of $b \in[0,50]$.

\begin{tabular}{|c|l|c|}
\hline$b$ & $\gamma_{b}$ & $b \gamma_{b}$ \\
\hline 0 & 3 & \\
1 & 1.564747 & 1.56475 \\
2 & 1.041362 & 2.08272 \\
3 & 0.776966 & 2.33090 \\
4 & 0.618679 & 2.47472 \\
5 & 0.513623 & 2.56812 \\
10 & 0.277208 & 2.77209 \\
15 & 0.189691 & 2.84537 \\
20 & 0.144151 & 2.88302 \\
25 & 0.116237 & 2.90593 \\
30 & 0.0973780 & 2.92134 \\
35 & 0.0837832 & 2.93241 \\
40 & 0.0735188 & 2.94075 \\
45 & 0.0654947 & 2.94726 \\
50 & 0.0590496 & 2.95248 \\
\hline
\end{tabular}


From this theorem, we can determine the values of the constant $\gamma_{b}$, see Table 1 .

It may be of interest to note that Table 1 does not result from straight numerical integration, which would be conducive to various numerical difficulties. Its derivation was first obtained instead by symbolic integration performed by the Maple system [3]. For values $1-10$ and 15(5)50, the computation took a little over 600 seconds of CPU time (on a Sun 3 machine performing $3 \cdot 10^{6}$ instructions per second and equipped with $12 \cdot 10^{6}$ bytes of memory). For instance, we have for $b=50$, the verbatim form of $\gamma_{b}$,

$$
\frac{3159614683170552814765839048751265660686349}{820545673826076765176005607309978880}-390150 \pi^{2} .
$$

The first symbolic values are given below.

\begin{tabular}{|r|l|}
\hline$b$ & $\gamma_{b}$ \\
\hline 0 & 3 \\
1 & $120-12 \pi^{2}$ \\
2 & $534-54 \pi^{2}$ \\
3 & $1422-144 \pi^{2}$ \\
4 & $\frac{5923}{2}-300 \pi^{2}$ \\
5 & $\frac{53301}{10}-540 \pi^{2}$ \\
10 & $\frac{252794897}{7056}-3630 \pi^{2}$ \\
\hline
\end{tabular}

All these numerical data suggest definite patterns: $\gamma_{b}$ is a rational function of $\pi$, the coefficient of $\pi^{2}$ has a simple form, and $\gamma_{b} \approx 3 / b$ for large $b$. In effect, we have:

THEOREM 2 (i). The coefficient $\gamma_{b}$ is a linear function of $\pi^{2}$,

$$
\frac{1}{3} \gamma_{b}=6 b^{2}+9 b+1-6 b(b+1)^{2}\left[\frac{\pi^{2}}{6}-\sum_{j=1}^{b} \frac{1}{j^{2}}\right] .
$$

(ii) Asymptotically, for large b, we have

$$
\frac{1}{3} \gamma_{b}=\frac{1}{b}-\frac{4}{5 b^{2}}+\frac{2}{5 b^{3}}+\frac{2}{35 b^{4}}-\frac{2}{7 b^{5}}+\frac{2}{35 b^{6}}+\frac{2}{5 b^{7}}-\frac{14}{55 b^{8}}+O\left(\frac{1}{b^{9}}\right) \text {. }
$$

On the practical side, we would like to comment on this $33 \%$ page filling ratio. Often, for a data structure, a relatively low filling ratio can be obviated by a suitable allocation policy. Assume for instance, that we choose to implement a paged quadtree structure which we design with a parameter $b=60$; the pages created are called "logical" pages. If we allocate physical pages of capacity $\beta=20$, the quadtree structure built with logical pages with parameter $b=60$ will have each of its logical

1 The absolute errors provided by the approximate formula obtained by dropping the $O($.) error terms are of order respectively $10^{-3}, 10^{-5}, 10^{-8}$ for $b=2,4,8$. 
pages spread over 1, 2, or 3 physical pages. Our analysis (see Section 4 and Theorem 5) enables us to quantify precisely what happens: In that situation, the number of disk accesses increases slightly and it is on an average 1.421145; in counterpart, the (physical) filling ratio improves appreciably and becomes close to 0.67273 . In summary we more than double the occupancy rate at the expense of an increase of about $40 \%$ of the access time.

The analysis techniques developed here are of some level of generality, since they apply to a fairly general class of additive cost measures on quadtrees. Theorem 4 discusses statistics on arbitrary node types in quadtrees; as a particular application, we are able to characterize the expected number of pages containing $k$ elements $(0 \leq k \leq b)$, and thus attain a precise evaluation of the page occupancy profile in paged quadtrees.

The evaluation of filling ratios is useful in order to assess and possibly optimize various allocation strategies. In this spirit, the paper concludes with a brief survey of analytical results available for index trees of various sorts.

To a large extent our Theorem 2 owes its existence to the integration capabilities of the Maple system for computer algebra [3] which revealed the unsuspected occurrence of closed form expressions involving dilogarithms and made it possible to carry out easily rather intensive computations.

\section{Paged quadtrees.}

Our data model assumes data in random order. Without loss of generality, we take them independently and uniformly distributed over the unit square $\mathscr{Q}=[0,1] \times[0,1]$. Given a sequence $S=\left(S_{1}, S_{2}, \ldots, S_{n}\right)$ of points, $S \in \mathscr{Q}^{n}$ we form a tree, called a $b$-quadtree, by the following rules:

- If $|S| \leq b$, then the tree consists of a single external (page) node that contains $S$ itself.

- If $|S|>b$, then the first element $S_{1}$ of $S$ partitions the other elements $\left(S_{2}, \ldots, S_{n}\right)$ into four subsequences, based on the four quadrants (North-West, North-East, etc.) determined by $S_{1}$, namely $S_{N W}, S_{N E}, S_{S W}, S_{S E}$. The tree associated to $S$ is composed of a root which contains $S_{1}$ and of the four subtrees formed recursively from the four subsequences $S_{N W}, S_{N E}, S_{S W}, S_{S E}$.

The standard quadtree of Finkel and Bentley appears when $b=0$, and one singles out the external empty nodes. A $b$-quadtree can be alternatively viewed as a standard quadtree in which maximal subtrees of size $\leq b$ are grouped into individual pages. With this view, the number of pages or the number of internal nodes of a paged $b$-quadtree are simple parameters of the underlying standard quadtree. Our paper is in fact a paper on cost measures on standard quadtrees applied to paging. Notations. Given a sequence of numbers $\left\{f_{n}\right\}_{n \geq 0}$, its generating function(GF) is 


$$
f(z)=\sum_{n \geq 0} f_{n} z^{n}
$$

We also use $\left[z^{n}\right] f(z)$ in order to represent the coefficient of $z^{n}$ in $f(z)$, that is $\left[z^{n}\right] f(z)=f_{n}$.

ADDITIVE FUNCTIONS OVER QUADTREES. We consider here a general additive function over standard quadtrees

$$
\begin{aligned}
& f[t]=e_{|t|}+\sum_{j=1}^{4} f\left[t_{j}\right] \\
& f[\emptyset]=e_{0},
\end{aligned}
$$

with $t_{1}, t_{2}, t_{3}, t_{4}$ the root subtrees of $t$; there $e_{n}$ is a sequence of numbers, called the "tolls". Thus $f[t]$ represents the total cost associated to a tree, when there is a toll (depending on subtree sizes) at each node in the tree.

For instance, if the toll is $e_{n} \equiv 1$, then $f[t]$ is the total number of nodes in the tree, $f[t]=|t|$; if $e_{n}=n$, we get the path length of the tree. Given the paging parameter $b$, the number of internal nodes in the associated $b$-quadtree corresponds clearly to the toll function

$$
e_{n}=1 \text { if } n>b ; \quad e_{n}=0 \text { if } 0 \leq n \leq b .
$$

In this case, the number of external nodes (i.e., pages) is $3 f[t]+1$, because of the general conservation law on quaternary trees.

In the sequel, we keep $f[t]$ in order to denote a generic tree cost, reserving $I[t]$ and $P[t]=3 I[t]+1$ for the number of internal and external nodes, when the parameter $b$ has been fixed.

If $f[$.$] is a cost, we let f_{n}$ be its expectation, when taken over all randomly built quadtrees over $n$ data items. The generating functions of the sequences $\left\{e_{n}\right\}$ and $\left\{f_{n}\right\}$ are thus

$$
e(z)=\sum_{n \geq 0} e_{n} z^{n} ; \quad f(z)=\sum_{n \geq 0} f_{n} z^{n}
$$

LEMMA 1. Let $\left\{e_{n}\right\}$ be a toll sequence with $e_{0}=0$; let $f_{n}$ be the expectation of the corresponding cost as defined by Eq. (4). Then the associated GF's $e(z)$ and $f(z)$ are related by

$$
f(z)=\frac{(1+2 z)}{(1-z)^{2}} \int_{0}^{z} \frac{(1-t)^{3}}{t(1+2 t)^{2}}\left[\int_{0}^{t} \frac{(1+2 v)}{(1-v)^{2}} E(v) d v\right] d t
$$

where $E(z)$ is the modified cost generating function,

$$
E(z)=\frac{d}{d z} z(1-z) \frac{d}{d z} e(z)
$$


PROOF. Let $\pi_{n, k}$ denote the probability that a quadtree of size $n$ has its first (e.g., $N W)$ subtree of size $k$. We have $[5,9,10,18]$

$$
\pi_{n, k}=\frac{1}{n} \sum_{l=k}^{n-1} \frac{1}{l+1}
$$

An informal interpretation is that each of the $n$ possibilities, $\{0,1, \ldots, n-1\}$, for the number of elements going to West is equally likely and has probability $1 / n$; if $l$ elements are located West of the root, then each value $K \in[0 \ldots l]$ of the number of elements residing North-West is equally likely and has probability $1 /(l+1)$. (We refer the sceptical reader to the cited publications for more convincing arguments!)

With this form of the $\pi_{n, k}$, the standard recurrence for costs is

$$
f_{n}=e_{n}+4 \sum_{k=0}^{n-1} \pi_{n, k} f_{k}
$$

where we have taken advantage of obvious symmetries.

Thus, if we go to the realm of generating functions, we find the integral equation that corresponds to (7),

$$
f(z)=e(z)+4 \int_{0}^{z}\left[\int_{0}^{t} f(u) \frac{d u}{1-u}\right] \frac{d t}{t(1-t)} .
$$

By differentiations, we get the equivalent differential equation,

$$
z(1-z) \frac{d^{2}}{d z^{2}} f(z)+(1-2 z) \frac{d}{d z} f(z)-\frac{4}{1-z} f(z)=E(z),
$$

where

$$
E(z)=\frac{d}{d z} z(1-z) \frac{d}{d z} e(z) .
$$

First, one looks at the homogeneous equation defined by setting $E(z)=0$ inside (9).

One method ${ }^{2}$ consists in solving this equation by reducing it to a degenerate hypergeometric equation, as was done for similar problems in $[9,10]$ : We look for an approximate solution of the form $(1-z)^{\alpha}$, obtain the indicial equation $\alpha^{2}-4=0$ so that $\alpha= \pm 2$, try a solution of the form $\hat{f}(z)(1-z)^{-2}$, and find that $\hat{f}$ satisfies the derived equation,

${ }^{2}$ It is interesting to note that the equation is now in principle solvable by general purpose algorithms that determine rational solutions to linear ODE's, see e.g. [2]. Some amount of human interaction is however still needed since we impose additional analyticity requirements around 0 . Also, the general reduction of a quadtree analysis to hypergeometric equations is an especially effective and general tool $[9,10]$, so that we have decided to reduce ourselves to this form instead of directly involking a deus ex machina formula, $\phi(z)=(1+2 z) /(1-z)^{2}$. 


$$
z(1-z) \frac{d^{2}}{d z^{2}} \hat{f}(z)+(1+2 z) \frac{d}{d z} \hat{f}(z)-2 \hat{f}(z)=0 .
$$

This equation can be matched against the standard hypergeometric equation [27],

$$
z(1-z) \frac{d^{2}}{d z^{2}} Y(z)+(c-(a+b+1) z) \frac{d}{d z} Y(z)-a b Y(z)=0,
$$

by taking $c=1, a=-1$, and $b=-2$. Thus, we get for $\hat{f}$ the special hypergeometric form

$$
\hat{f}(z)={ }_{2} F_{1}[-1,-2 ; 1 ; z]=1+2 z .
$$

The whole process thus provides us with the particular solution to the homogeneous associate of (9),

$$
\phi(z)=\frac{\hat{f}(z)}{(1-z)^{2}}=\frac{1+2 z}{(1-z)^{2}} \quad \text { when } \quad E(z)=0,
$$

another independent solution being discarded as it has a logarithmic singularity at 0 .

Returning then to the inhomogeneous equation, we proceed by the "variationof-constant" method. We seek a solution of the form $\lambda(z) \cdot \phi(z)=\lambda(z)(1+2 z)$ / $(1-z)^{2}$. By construction, $\lambda^{\prime}(z)$ satisfies an ODE of order 1 , hence, we recover the solution to the original equation by two quadratures, the result being as stated above.

PAGING. If we specialize to the case of the number of pages in a $b$-quadtree, we get:

Lemma 2. The generating function for the expected number of pages in $a b$ quadtree is

$$
P(z)=\frac{1}{1-z}+\frac{3(1+2 z)}{(1-z)^{2}} \int_{0}^{z} \frac{(1-t)^{3}}{t(1+2 t)^{2}}\left[\int_{0}^{t} \frac{(1+2 v)}{(1-v)^{2}} E(v) d v\right] d t
$$

with

$$
E(z) \equiv E_{b}(z)=z^{b} \frac{1+b(1-z)+b(b+1)(1-z)^{2}}{(1-z)^{2}} .
$$

Proof. This is a simple application of the previous lemma. The tolls for the number of internal nodes in a $b$-quadtree are the $e_{n}$ given above (5), with GF equal to

$$
e(z)=\frac{z^{b+1}}{1-z} \text { and } E(z)=\frac{d}{d z} z(1-z) \frac{d}{d z} \frac{z^{b+1}}{1-z} .
$$

We derive in this way $I(z)$ by Lemma 1 . By the conservation law of quaternary trees, we finally have $P(z)=3 I(z)+1 /(1-z)$. 
This expression would in principle enable us to express in "closed form" the average number of pages (see $[5,18]$ for related computations done independently via a recurrence approach). We prefer however a direct route to asymptotics based on the usual method of singularity analysis [11].

SINGULARITY ANALYSIS. The general principle is that the asymptotic behaviour of coefficients $\left[z^{n}\right] P(z)$ can be determined from the asymptotic form of the function $P(z)$ around its dominant singularities. The conditions are based on analytic continuation. They make it possible to transfer on a term-by-term basis from asymptotic elements of $P(z)$ to matching asymptotic elements of $\left[z^{n}\right] P(z)$.

Here, from either the differential equation and general theorems [26], or more explicitly from the integral representations, we see that $P(z)$ has a unique isolated logarithmic singularity at $z=1$. Thus $P(z)$ is analytically continuable outside its circle of convergence, say in $|z|<2, \quad|\operatorname{Arg}(z-1)|>\pi / 4$. Also, from the integral representation, there results that, in this region,

$$
P(z)=\gamma_{b} /(1-z)^{2}+O\left((1-z)^{-1} \log (1-z)^{-1}\right) \quad(z \rightarrow 1) .
$$

By the techniques of singularity analysis, this local expansion together with the analytic continuation of $P(z)$ outside its circle of convergence are enough to make legal the term-by-term transfer to coefficients, namely

$$
P_{n}=\gamma_{b} \cdot n+O(\log n)
$$

This therefore completes the proof of Theorem 1.

LEAVES IN QUADTREES. In order to shed some light on the internals of the computation, we examine the determination of the expected number of leaves in a randomly grown quadtree. In that case, we have $b=1$, and look at internal nodes. With our earlier notations, the corresponding GF is $I(z)$; the expected number of leaves is then $n-\left[z^{n}\right] I(z)$.

The interest of the computations that follow is to introduce a special function, namely the dilogarithm.

For $b=1$, the function $E(z)$ is equal to $-1+2 z+(1-z)^{-2}$. The inner integral $\int_{0}^{t} \ldots d v$ in $(10)$ is then found to be

$$
-\frac{t(t-2)\left(4 t^{2}-7 t+4\right)}{(1-t)^{3}}+8 \log (1-t)
$$

Multiplying by $(1-t)^{3} t^{-1}(1+2 t)^{-2}$, and integrating, we find a sum of two terms, one corresponding to the rational part, the other to the logarithm. The part corresponding to the rational term is a standard elementary function.

Recall the definition of the dilogarithm as 


$$
\operatorname{Li}_{2}(z)=\int_{0}^{z} \log (1-t)^{-1} \frac{d t}{t}=\sum_{k=1}^{\infty} \frac{z^{k}}{k^{2}}
$$

(We refer the reader to Lewin's classic treatise for a full exposition of the theory of the dilogarithm [20] or to Berndt's review of its main properties in [1, Chap. 9].) A dilogarithm arises from integration of the logarithmic term, $8 \log (1-t)$, multiplied by the element $1 / t$ that comes from the partial fraction decomposition

$$
\frac{(1-t)^{3}}{t(1+2 t)^{2}}=\frac{1}{t}-\frac{1}{4}-\frac{27}{4(1+2 t)^{2}} \text {. }
$$

All computations done, we get

COROLLARY 3. The generating function for the number of non-leave nodes in a randomly grown quadtree $(b=1)$ is

$$
I(z)=\frac{z\left(28+13 z-z^{2}\right)}{(1-z)^{2}}+\frac{20+4 z}{1-z} \log (1-z)-8 \frac{1+2 z}{(1-z)^{2}} \operatorname{Li}_{2}(z),
$$

with $\mathrm{Li}_{2}(u)$ the dilogarithm function. Thus,

$$
\left[z^{n}\right] I(z)=\left(40-4 \pi^{2}\right) n+13-\frac{4}{3} \pi^{2}+\frac{4}{3 n^{3}}-\frac{4}{5 n^{4}}-\frac{4}{15 n^{5}}+\frac{4}{7 n^{6}}+\frac{4}{21 n^{7}}+O\left(\frac{1}{n^{8}}\right) .
$$

In particular, the proportion of leaves in a random quadtree of size $n$ is asymptotically $4 \pi^{2}-39=0.47841762$.

ProOF. (Sketch) Here we obtain directly the asymptotic form $I_{n} \sim \gamma_{1}^{*} n$, with $\gamma_{1}^{*}=\lim _{z \rightarrow 1}(1-z)^{2} I(z)=40-4 \pi^{2}$. (We also have $\gamma_{1}^{*}=\gamma_{1} / 3$ in terms of our standard notations.) The result for leaves follows by complementation to $n$ of the number of non-leaves.

An entirely similar process applies to the problem of estimating the number of pages for an arbitrary $b$. The occurrence of the dilogarithm which satisfies

$$
\operatorname{Li}_{2}(1)=\int_{0}^{1} \log (1-t)^{-1} \frac{d t}{t}=\sum_{k=1}^{\infty} \frac{1}{k^{2}} \equiv \frac{\pi^{2}}{6}
$$

"explains" the presence of $\pi^{2}$ in the explicit forms of $\gamma_{b}$ given in the introduction. We shall see that such a treatment can be extended to arbitrary node types.

From the exact form of $I(z)$, we also observe that the coefficient $\left[z^{n}\right] I(z)$ is expressible in terms of the harmonic number $\zeta_{n}(1)$ and the generalized harmonic number $\zeta_{n}(2)$, where

$$
\zeta_{n}(s)=\sum_{k=1}^{n} \frac{1}{k^{s}}
$$


Such expressions were obtained by Laforest et al. $[17,18]$ using a direct theory of quadtree recurrences from [5] which constitutes an alternative to our Lemma 1. A fine result of [17] is.that the proportion of nodes with one non-empty subtree is $(24 \zeta(3)-156 \zeta(2)+228)=0.239651$.

We are going to elicit the finer structure of $\gamma_{b}$ as a function of $b$ in the next section.

\section{The occupancy constants $\gamma_{b}$.}

Our approach now consists in computing the generating function of the numbers $\gamma_{b}$. The following lemma provides a more direct access to the numbers $\gamma_{b}$ that avoids integration, and also proves that $\gamma_{b}$ has a rational expression in terms of $\pi^{2}$. Analysing the singularity of the GF of the $\gamma_{b}$ further provides detailed asymptotic informations on these coefficients.

LEMMA 3. The generating function $\gamma(u)$ of the numbers $\gamma_{b}$ defined by $\gamma(u)=\sum_{b=0}^{\infty} \gamma_{b} u^{b}$ is given by

$$
\begin{aligned}
\gamma(u)= & \frac{3}{(1-u)^{4}} \cdot\left[\left(-4 u-2 u^{2}\right) \pi^{2}+\left(1+30 u-27 u^{2}-4 u^{3}\right)\right. \\
& \left.+\left(-6-24 u+30 u^{2}\right) \log (1-u)+\left(24 u+12 u^{2}\right) \operatorname{Li}_{2}(u)\right] .
\end{aligned}
$$

Proof. Define the basic integrals

$$
J_{\alpha}(u)=\int_{0}^{1}\left[\int_{0}^{t} \frac{(1+2 v)}{(1-v)^{4-\alpha}} \frac{d v}{(1-u v)}\right] \frac{(1-t)^{3}}{t(1+2 t)^{2}} d t .
$$

These serve as the basis in which to express the generating function $\gamma(u)$. From the summations

$$
\sum_{m} u^{m} v^{m}=\frac{1}{1-u v}, \quad \sum_{m} m \cdot u^{m} v^{m}=u \frac{d}{d u} \frac{1}{1-u v}, \quad \sum_{m} m(m-1) \cdot u^{m} v^{m}=u^{2} \frac{d^{2}}{d u^{2}} \frac{1}{1-u v},
$$

and the integral representation of $\gamma_{b}$, we find that

$$
\frac{1}{3} \gamma(u)=J_{0}(u)+u \frac{d}{d u} J_{1}(u)+u^{2} \frac{d^{2}}{d u^{2}} J_{2}(u)+2 u \frac{d}{d u} J_{2}(u) .
$$

Our problem is thus reduced to computing the quantities $J_{0}, J_{1}, J_{2}$.

In principle, the problem resembles the computation in our earlier section; see for instance the particular case of counting leaves. It is however complicated by the extra factor $(1-u v)^{-1}$ that introduces an additional singularity in the computations.

Preliminary investigations performed with the Maple system first revealed the possibility of an explicit solution that involves dilogarithms. Once this has been 
recognized, it is possible to carry out the double integration. Minor computer algebra difficulties arise from several sources: certain normal forms provided by integration routines sometimes introduce transformations of the form $\log (1-t) \mapsto \log (t-1)+\log (-1)$; the solutions, though representing generating functions, may have apparent singularities at 0 that need to be eliminated; finally, some of the expressions obtained involve the dilogarithm under a form that is singular at 0 .

We dispense ourselves from giving here all the explicit forms of the $J_{\alpha}$ and the partial integrals involved. Once found by whatever means, they are all that is needed in order to reconstruct a complete proof of the expression given for $\gamma(u)$, since the correctness of integrals can always be established by differentiation. We briefly discuss in the Appendix the sequence of steps neded to obtain $\gamma(u)$ using the Maple system.

In passing, the solution there is expressed in terms of Maple's version of the dilogarithm function

$$
\operatorname{dil} \log (u)=\operatorname{Li}_{2}(1-u)=\sum_{k=1}^{\infty} \frac{(1-u)^{k}}{k^{2}} .
$$

The reduction to a standard dilogarithm, evaluated near 0 , is achieved via the well known transformation formula (whose proof is a single integration by parts):

$$
\operatorname{Li}_{2}(1-z)+\operatorname{Li}_{2}(z)=\frac{\pi^{2}}{6}-\log z \log (1-z) .
$$

From this, the proof of the lemma follows.

From Lemma 3, explicit forms of the $\gamma_{b}$ are derived. The principle is to express the GF $\gamma(u)$ in the basis of functions

$$
h_{1, j}(u)=\bar{\theta}^{j} \frac{\log (1-u)^{-1}}{(1-u)} \text { and } h_{2, j}(u)=\bar{\theta}^{j} \frac{\mathrm{Li}_{2}(u)}{(1-u)},
$$

where $\bar{\theta}$ represents the differential operator $\bar{\theta}\{f(u)\} \equiv \frac{d}{d u}\{u f(u)\}$, the coefficients of these functions involving generalized harmonic numbers, since

$$
\frac{1}{1-u} \operatorname{Li}_{2}(u)=\sum_{n=0}^{\infty} \zeta_{n}(2) u^{n}
$$

We find

$$
\frac{1}{3} \gamma(u)=6\left[\bar{\theta}^{3}-\bar{\theta}^{2}\right]\left\{\frac{1}{1-u} \operatorname{Li}_{2}(u)\right\}+\frac{1+13 u-2 u^{2}}{(1-u)^{3}}-2 \pi^{2} \frac{u(2+u)}{(1-u)^{4}}
$$

It is an easy matter to expand $\gamma(u)$ from this form. This completes the proof of Part (i) of Theorem 2. 
The asymptotic form of $\gamma_{b}$ next results from singularity analysis. There is a full asymptotic expansion of $\gamma(u)$ around $u=1$. The term $\mathrm{Li}_{2}(u)$ is expanded using the basic functional equation (13). In this way, we find

$$
\frac{1}{3} y(u)=\left[\mathrm{Li}_{1}(u)+\frac{1}{12}\right]+(1-u)\left[\frac{4}{3} \operatorname{Li}_{1}(u)+\frac{17}{75}\right]+(1-u)^{2}\left[\frac{3}{5} \operatorname{Li}_{1}(u)+\frac{17}{100}\right]+\ldots
$$

where $\operatorname{Li}_{1}(u)=\log (1-u)^{-1}$. Using the identity

$$
\left[u^{m}\right](1-u)^{k} \operatorname{Li}_{1}(u)=\frac{(-1)^{k} k !}{m(m-1)(m-2) \ldots(m-k)},
$$

we map the singular expansion (15) into a matching expansion for $\gamma_{m}=\left[u^{m}\right] \gamma(u)$, the conditions of analytic continuation being clearly satisfied here. In this way, we get

$$
\frac{1}{3} \gamma_{m}=\frac{1}{m}-\frac{4}{5} \cdot \frac{1 !}{m(m-1)}+\frac{3}{5} \cdot \frac{2 !}{m(m-1)(m-2)}-\ldots,
$$

which can be normalized into a standard expansion in descending powers of $1 / m$. This completes the proof of Part (ii) of Theorem 2.

\section{Node types.}

The same methods make it possible to analyze the number of occurrences of nodes of arbitrary composition in quadtrees. Assume we look for the expected number of nodes $v$ in a random tree of size $n$ such that the subtree rooted at $v$ has a fixed shape $\omega$. This corresponds to a toll sequence $\hat{e}_{n}$ such that $\hat{e}_{n}=0$ for all values of $n \neq|\omega|$. For $p=|\omega|, \hat{e}_{p}$ is a rational number $\varepsilon_{\omega}$ equal to the probability that the tree shape $\omega$ occurs as a randomly built quadtree on $p$ elements. That probability is computable inductively over subtrees using the form of splitting probabilities [9]

$$
\pi_{n_{1}, n_{2}, n_{3}, n_{4}}=\frac{1}{n \cdot n !} \frac{\left(n_{1}+n_{2}\right) !\left(n_{3}+n_{4}\right) !\left(n_{1}+n_{3}\right) !\left(n_{2}+n_{4}\right) !}{n_{1} ! n_{2} ! n_{3} ! n_{4} !},
$$

which represents the probability that the $(N W, N E, S W, S E)$ root subtrees have sizes $n_{1}, n_{2}, n_{3}, n_{4}$, respectively. If $\omega=\left\langle r ; t_{1}, t_{2}, t_{3}, t_{4}\right\rangle$ is a tree with root $r$ and $t_{j}$ as root subtrees, we have

$$
\varepsilon_{\omega}=\pi_{\left|t_{1}\right|,\left|t_{2}\right|,\left|t_{3}\right|,\left|t_{4}\right|} \cdot \varepsilon_{\omega_{1}} \varepsilon_{\omega_{2}} \varepsilon_{\omega_{3}} \varepsilon_{\omega_{4}}
$$

together with the initial condition $\varepsilon_{\omega}=1$ if $|\omega| \leq 1$.

Thus, we find the toll generating function $\hat{e}(z)=\varepsilon_{\omega} z^{p}$, with $p=|\omega|$, where $\varepsilon_{\omega}$ is an easily computable rational number. If we compare this to the toll GF considered earlier in connection with paging, $e_{b}(z)=z^{b+1} /(1-z)$, we see that

$$
\hat{e}(z)=\varepsilon_{\omega}\left[e_{|\omega|-1}(z)-e_{|\omega|}(z)\right] .
$$

By linearity of the cost transform (Lemma 1), we get: 
THEOREM 4. Consider an arbitrary node type defined by a tree shape $\omega$. The expected number of nodes of type $\omega$ admits the asymptotic form

$$
\left(n \varepsilon_{\omega} / 3\right)\left[\gamma_{|\omega|-1}-\gamma_{|\omega|}\right],
$$

where $\varepsilon_{\omega} \in \mathbb{Q}$ is the probability of tree shape $\omega$ amongst all quadtrees of size $|\omega|$.

The coefficients are therefore $\mathbb{Q}$-linear combinations of 1 and $\pi^{2}$.

This generalizes results of Laforest et al. $[17,18]$ who studied nodes having a single child. (Full asymptotic expansions for the number of nodes of a given type could also be obtained in the style of Corollary 3.) As a check, we can also retrieve the expected number of leaves, corresponding to $|\omega|=1$, which leads to the asymptotic form $(n / 3)\left(\gamma_{0}-\gamma_{1}\right)$.

The $\gamma_{b}$ thus appear as fundamental constants in the analysis of quadtrees. From them, one can determine the profile of page occupancy.

THEOREM 5. In a paged b-quadtree, the expected number of pages containing $k$ elements, $0 \leq k \leq b$, is of the asymptotic form $\gamma_{b, k} \cdot n$, with

$$
\gamma_{b, k}=\frac{\gamma_{b}}{b+1}+B\left[H_{b+1}-1-H_{k}\right], \quad B=\frac{2}{3} \cdot \frac{3 b \gamma_{b}+2 \gamma_{b}-6}{b(b+1)},
$$

where $H_{n} \equiv \zeta_{n}(1)=1+\frac{1}{2}+\ldots+\frac{1}{n}$ is the standard harmonic number.

Proof. As an application of Theorem 4, we first count the expected number of pages that satisfy the conditions: (i) they are a leftmost child; (ii) they contain $k$ elements; (iii) their father is the root of a subtree with $m$ elements for some fixed $m>b$. Using the form of the splitting probabilities $\pi_{m, k}=\left(H_{m}-H_{k}\right) / m$, we find that the asymptotic proportion of such pages is

$$
(3 m)^{-1}\left(H_{m}-H_{k}\right)\left[\gamma_{m-1}-\gamma_{m}\right] \text {. }
$$

The constant $\gamma_{b, k}$ is obtained by multiplying by 4 (to take care of all four child nodes) and summing over all values of $m$ from $b+1$ to $\infty$. In this way, we see that

$$
\gamma_{b, k}=A-B H_{k}, A=\sum_{m=b+1}^{\infty} \frac{4 H_{m}}{3 m}\left[\gamma_{m-1}-\gamma_{m}\right], B=\sum_{m=b+1}^{\infty} \frac{4}{3 m}\left[\gamma_{m-1}-\gamma_{m}\right]
$$

The constants $A, B$ could probably be found by direct summation. However, it is simpler, once their existence has been recognized, to identify them by means of conservation laws for nodes. We have

$$
\sum_{k=0}^{b} \gamma_{b, k}=\gamma_{b} \text { and } \sum_{k=0}^{b} k \cdot \gamma_{b, k}=1-\frac{\gamma_{b}}{3} .
$$

The first relation expresses that a page contains a certain number $k$ of elements for 
some $k \in[0 . . b]$; the second relation consists in estimating the proportion of elements contained in pages either as non-internal elements (whose proportions is $\left.1-\gamma_{b} / 3\right)$ or based on the size of the page that contains them.

We use the easy relations

$$
\sum_{k \leq b} H_{k}=(b+1)\left(H_{b+1}-1\right), \quad \sum_{k \leq b} k H_{k}=\frac{1}{2} b(b+1) H_{b+1}-\frac{1}{4} b(b+1),
$$

and then solve for $A$ and $B$ the system (17). In this way, we obtain the values of $A$, $B$ and the statement of the theorem follows.

For instance for $b=10$, we find the following proportions

$$
\begin{aligned}
& \gamma_{10,0}=0.06034, \quad \gamma_{10,1}=0.04294, \quad \gamma_{10,2}=0.03424, \quad \gamma_{10,3}=0.02844 \text {, } \\
& \gamma_{10,4}=0.02409, \quad \gamma_{10,5}=0.02061, \quad \gamma_{10,6}=0.01771, \quad \gamma_{10,7}=0.01523 \text {, } \\
& \gamma_{10,8}=0,01305, \quad \gamma_{10,9}=0.01112, \quad \gamma_{10,10}=0.00938 \text {. }
\end{aligned}
$$

All these constants have again exact forms that are expressible as functions of $\pi^{2}$. It is from them that we can analyze arbitrary page allocation strategies; see the example given in the introduction with $b=60, \beta=20$ and the corresponding Figure 1.

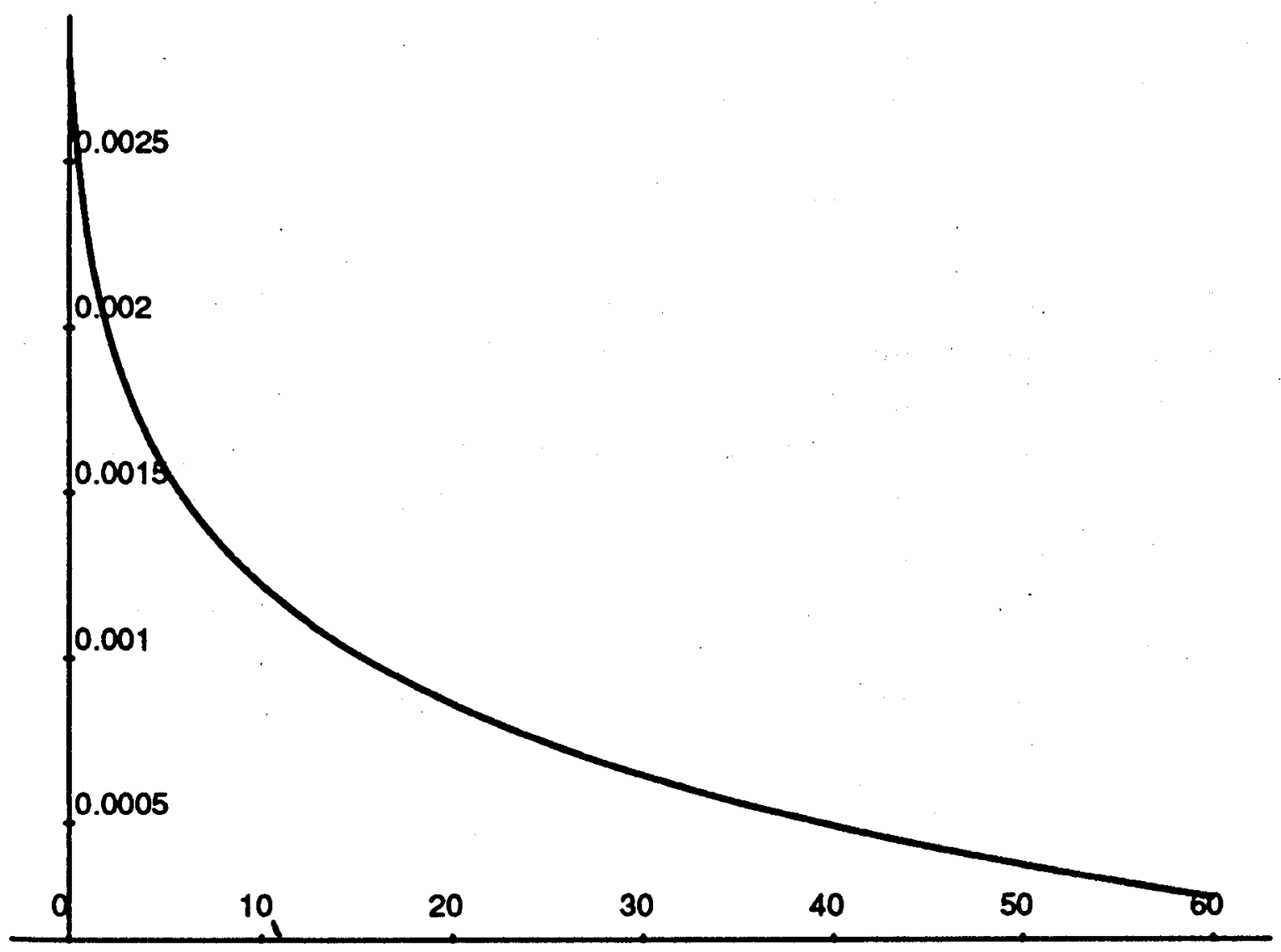

Fig. 1. The sequence of constants $\gamma_{b, k}$, when $b=60$. For instance, the total number of pages is about $0.049 n \approx n / 20$ since $\gamma_{60}=0.04933$. The number of empty pages is about $0.0028 n \approx n / 355$ and the number of full pages is about $0.00027 n \approx n / 3636$, so that roughly $n / 60$ records in a tree are to be found in a full page. 


\section{Conclusions.}

We conclude this paper with a brief overview of some major algorithms for maintaining dynamic tree structures in a paging environment. There are two major categories since structures are built either based on order properties of the data - the comparison based data structures - or on digital properties. Some of the trees are of fixed degree ( 2 or 4 depending on the dimension of the data space: binary search trees, tries, quadtrees, etc.); others have a branching degree that varies with $b$ (e.g., for $B$-trees it varies between $b / 2$ and $b$; for $m$-ary search trees, it is equal to $m$ with $m=b+1$, etc.). We refer to either Sedgewick's book [24] or to Gonnet's encyclopedia [14] as general sources on the algorithmic aspects. Average case analysis techniques are reviewed in [25], and tree models in [29].

Each analysis of storage occupancy normally poses an interesting mathematical problem. In this quick review, we also mention the major mathematical techniques at stake.

COMPARISON-BASED STRUCTURES. Binary search trees [16, Sec. 6.2.2] are the simplest structures to analyse. We consider the strategy already discussed for quadtrees whereby a maximal subtree of size $\leq b$ is stored into a single page. It is then found that the expected number of pages is asymptotic to $2 n /(b+2)$. In other words, storage occupancy is near $50 \%$. The generating function equations are simpler in this case. The main equation is of the form

$$
f(z)=e(z)+2 \int_{0}^{z} f(t) \frac{d t}{1-t} .
$$

This reduces to a differential equation of order 1 that can be solved by quadratures. Many parameters can be analyzed in this way by varying the "toll" GF. The model is the same as the one underlying Quicksort, see Knuth's book [16, p. 121] and Hennequin's thesis [15]. In particular, we find that the number of pages containing $r$ elements is $\sim 2 n /((b+1)(b+2))$ for $r \in[0 \ldots b]$ : In other words, pages with filling type $0 / b, 1 / b, \ldots, b / b$ are all equally frequent.

The storage occupancy of search trees whose degree is $m=b+1$ (a node contains $b$ keys and $b+1$ pointers) is investigated extensively by Mahmoud and Pittel [21]. The cost generating function satisfies a linear differential equation of order $b$, namely

$$
\frac{d^{b}}{d z^{b}} f(z)=e(z)+\frac{(b+1) !}{(1-z)^{b}} f(z) .
$$

The analysis is made possible because there is a regular singularity at $z=1$. It is found (see also [16, Ex. 6.2.4.10]) that the number of nodes in the tree is on average

$$
\sim \frac{n}{2\left(H_{b+1}-1\right)} \text { with } H_{m} \text { a harmonic number. }
$$


Storage utilization tends to 0 as $b$ gets large! In fact, Mahmoud and Pittel obtain asymptotic distribution results, a rather remarkable fact, since this requires analyzing a non-linear difference differential equation of high order.

The efficiency of $m$-ary search trees $(m=b+1)$ gets quite low as $b$ becomes large. However, balancing is a good solution with guaranteed worst case performance (at worst $50 \%$ ). Yao [28] has shown that for $B$-trees of large order that are balanced the storage occupancy rate approaches $\log 2$, the number of nodes being approximately $\approx n / b \log 2$. A number of variations to multiway trees have been proposed, for instance Poblete and Cunto's [4], and we redirect the reader to [14] for an extensive bibliography.

Yao's paper [28] is well known as the source of so-called fringe analyses that are based on Markovian approximations and matrix analysis. Mathematically, our results regarding quadtrees have been based on an integral transform (Lemma 1) that permits to resolve algebraically a wide class of cost functions on quadtrees; they further rely on singularity analysis and on special functions (the hypergeometric equation, the dilogarithm). Quite clearly, the approach taken here is general and applies to almost any conceivable additive parameters on quadtrees.

Digital METHODs. Digital methods use a separation principle based on bits of records (or their hashed values). The paging of small subfiles is analyzed by Knuth using methods partly suggested by de Bruijn, see Section 6.3 of [16] and the methods of pages $131 \mathrm{ff}$. there. The equations are difference equations of the form

$$
f(z)=e(z)+2 e^{z / 2} f(z / 2)
$$

The treatment relies on iteration and Mellin transforms. The number of pages in a trie involves some small oscillating terms, and neglecting them, it can be approximated by $n /(b \log 2)$, refer to Exercice 6.3.20 of [16], and read between the lines. The analysis is also relevant to dynamic hashing schemes $[6,19]$. The same analytic principles apply to quadtries whose evaluation is isomorphic to that of $m$-ary tries for $m=4$.

The digital tree structure can be extended by letting nodes contain up to $b$ elements, but still retaining the binary branching principle. The corresponding equation becomes a difference-differential equation

$$
\frac{d^{b}}{d z^{b}} f(z)=e(z)+2 e^{z / 2} f(z / 2)
$$

Mellin transforms and singularity analysis are the main ingredients of that analysis. Apart from fluctuations, the number of pages is found [13] to be of the form $n /(b \log 2)$. Thus, the ratio of $69 \%$ strikes again here.

For completeness, we have also tabulated some of the formulae for extendible hashing and grid files access methods. They concern the size of the directory which exhibits a non-linear growth of the form $n^{\beta}, \beta>1$. However, the non-linearity factor 


\begin{tabular}{|c|c|c|}
\hline & Comparison based & Digital \\
\hline \multirow[t]{4}{*}{ 1-dim } & $\begin{array}{l}\text { Binary search tree, }[0.5] \\
\qquad 2 n /(b+2)\end{array}$ & $\begin{array}{l}\text { Binary digital trie, [0.69] } \\
n /(b \log 2)\end{array}$ \\
\hline & $\begin{array}{c}m \text {-ary search tree, }[0.0] \\
(m=b+1): \quad n /\left(2\left(H_{m}-1\right)\right)\end{array}$ & $\begin{array}{c}\text { Paged } b \text {-digital tree, [0.69] } \\
\approx n /(b \log 2)\end{array}$ \\
\hline & $\begin{array}{c}\text { Balanced } B \text {-tree, }[0.69] \\
n /(b \log 2)\end{array}$ & \\
\hline & & $\begin{array}{l}\text { Extendible Hash directory, [0.0] } \\
\qquad \approx 4 b^{-1} n^{1+1 / b}\end{array}$ \\
\hline \multirow[t]{2}{*}{ 2-dim } & $\begin{array}{l}\text { Quadtree, }[0.33] \\
\approx \approx 3 n / b\end{array}$ & $\begin{array}{l}\text { Quadtrie, }[0.46] \\
3 n /(2 b \log 2)\end{array}$ \\
\hline & & $\begin{array}{l}\text { Grid file directory, [0.0] } \\
\approx n^{1+1 /(2 b+1)}\end{array}$ \\
\hline
\end{tabular}

Fig. 2. A summary of some major paging strategies for trees and their expected performance in asymptotic form. There $n$ is the file size, and $b$ represents the page capacity in terms of records that a page can contain. The number in brackets, [p], represents a numerical approximation of the filling ratio $\rho$ such that the expected storage occupancy varies like $n /(b \rho)$.

is of the rough form $n^{1 / b}$, so that the observed behaviour is practically linear provided small values of $b$ are avoided. The estimates are due to Flajolet [8] and Régnier [22]. They are based on occupancy statistics, saddle point estimates and Mellin transforms.

Results in this paper indicate that, under paging conditions, trees of low degree (binary search trees and tries, quadtrees and quadtries, generalized digital trees) compare very favorably to trees with high branching degree, except when balancing can be maintained. A variety of methods from discrete mathematics have surfaced in the analysis of storage occupancy for tree data structures. The methods employed here constitute yet another illustration of the power of differential equations in conjunction with singularity analysis techniques in the area of the average case analysis of algorithms which were introduced in [12].

\section{Acknowledgements.}

The authors would like to acknowledge the constant help and support of the Maple system that might well have been a co-author of the paper. By suggesting the existence of closed form in terms of dilogarithms, Maple made it possible for us to ultimately derive Theorems 2,4 , and 5 in a clean form.

Mamoru Hoshi would like to express his gratitude to the members of the Algorithms Project at INRIA for their encouragements. 


\section{REFERENCES}

1. Berndt, B. C. Ramanujan's Notebooks, Part I. Springer Verlag, 1985.

2. Bronstein, M. On solutions of linear ordinary differential equations in their coefficient field. Tech. Rep. 152, Department Informatik, ETH, January 1991.

3. Char, B. W., Geddes, K. O., Gonnet, G. H., Monagan, M. B. and Watt, S. M. MAPLE: Reference Manual. University of Waterloo, 1988. Sth edition.

4. Cunto, W. and Poblete, P. Transforming multiway trees into a practical external data structure. Acta Informatica 26, 3 (1988), 193-212.

5. Devroye, L. and Laforest, L. An analysis of random d-dimensional quad trees. SIAM Journal on Computing 19 (1990), 821-832.

6. Fagin, R., Nievergelt, J., Pippenger, N. and Strong, R. Extendible hashing: A fast access method for dynamic files. A.C.M. Trans. Database Syst. 4 (1979), 315-344.

7. Finkel, R. A. and Bentley, J. L. Quad trees, a data structure for retrieval on composite keys. Acta Informatica 4 (1974), 1-9.

8. Flajolet, P. On the performance evaluation of extendible hashing and trie searching. Acta Inf. 20(1983), 345-369.

9. Flajolet, P., Gonnet, G., Puech, C. and Robson, J. M. The analysis of multidimensional searching in quad-trees. In Proceedings of the Second Annual ACM-SIAM Symposium on Discrete Algorithms (Philadelphia, 1991), SIAM Press, pp. 100-109.

0. Flajolet, P., Gonnet, G., Puech, C. and Robson, J. M. Analytic variations on quadtrees. Algorithmica (1992). 24 pages, to appear.

1. Flajolet, P. and Odlyzko, A. M. Singularity analysis of generating functions. SIAM Journal on Discrete Mathematics 3, 2 (1990), 216-240.

2. Flajolet, P. and Puech, C. Partial match retrieval of multidimensional data. Journal of the ACM 33, 2 (1986), 371-407.

3. Flajolet, $P$. and Richmond, B. Generalized digital trees and their difference-differential equations, Apr. 1991. 15 pages. INRIA Research Report, in press. Also submitted to Random Structures and Algorithms.

4. Gonnet, G. H. and Baeza-Yates, R. Handbook of Algorithms and Data Structures: in Pascal and C, second ed. Addison-Wesley, 1991.

5. Hennequin, P. Analyse en moyenne d'algorithmes, tri rapide et arbres de recherche. $\mathrm{PhD}$ thesis, École Polytechnique 1991.

6. Knuth, D. E. The Art of Computer Programming, vol. 3: Sorting and Searching. Addison-Wesley, 1973.

7. Labelle, G. and Laforest, L. Variations combinatoires autour des arborescences hyperquaternaires. Tech. rep., LACIM, UQAM, Montreal, November 1991.

8. Laforest, L. Étude des arbres hyperquaternaires. Tech. Rep. 3, LACIM, UQAM, Montreal, Nov. 1990. (Author's PhD Thesis at McGill University).

9. Larson, P. A. Dynamic hashing. BIT 18 (1978), 184-201.

:0. Lewin, L. Polylogarithms and Associated Functions. North-Holland, New York, 1981.

1. Mahmoud, H. M. and Pittel, B. Analysis of the space of search trees under the random insertion algorithm. J. Algorithms 10 (1989), 52-75.

2. Régnier, M. Analysis of grid file algorithms. BIT 25 (1985), 335-357.

:3. Samet, H. The Design and Analysis of Special Data Structures. Addison-Wesley, 1990.

:4. Sedgewick, R. Algorithms, second ed. Addison-Wesley, Reading, Mass., 1988.

5. Vitter, J. S. and Flajolet, P. Analysis of algorithms and data structures. In Handbook of Theoretical Computer Science, J. van Leeuwen, Ed., vol. A: Algorithms and Complexity. North Holland, 1990, ch. 9, pp. 431-524.

16. Wasow, W. Asymptotic Expansions for Ordinary Differential Equations. Dover, 1987. A reprint of the John Wiley edition, 1965.

7. Whittaker, E. T. and Watson, G. N. A Course of Modern Analysis, fourth ed. Cambridge University Press, 1927. Reprinted 1973.

8. Yao, A. C.-C. On random 2-3 trees. Acta Informatica 9, 2 (1978), 159-170.

'9. Mahmoud, H. M. Evolution of Random Search Trees, Wiley, 1992. 


\section{APPENDIX}

\section{Computation of $\gamma(u)$.}

We indicate here the sequence of steps needed to obtain the generating function $\gamma(u)$ of the paging constants $\gamma_{b}$. The final form involving a dilogarithm is the subject of Lemma 3, and it is from there that the explicit form of $\gamma_{b}$ stated in Theorem 2 follows.

The computation by hand would be rather horrendous. The computation was performed with the help of the Maple system, and it involves on the part of the system fairly non trivial integration and simplification capablities.

We concentrate here on the determination of the function $J_{0}(u)$ defined in the text. What is needed is a double integral. Set

$$
I_{0}(t ; u)=\int_{0}^{t} \frac{(1+2 v)}{(1-v)^{4}} \frac{d v}{1-u v} \text { and } I_{1}(z ; u)=\int_{0}^{z} \frac{(1-t)^{3}}{t(1+2 t)^{2}} I_{0}(t ; u) d t .
$$

First the integral $I_{0}(t ; u)$ is computed as the primitive function of a rational function. The result involves a $\mathbb{Q}(z, u)$ rational form in various logarithms, and its length as provided by the Maple system is 738 . Another level of integration yields $I_{1}(z ; u)$ : the resulting expression involves logarithms and dilogarithms,

$$
\log (z), \quad \log (u), \quad \log (1-z), \quad \log (1-u z), \quad \log (1+2 z), \quad \operatorname{Li}_{2}(z), \quad \operatorname{Li}_{2}(u z) .
$$

The resulting expression is quite large, having size 1995.

The next step consists in determining $J_{0}(u)$ as $\lim _{z \rightarrow 1} I_{1}(z ; u)$. This is achieved via the use of the limit function which "knows" properties of the dilogarithm, like $\mathrm{Li}_{2}(1)=\pi^{2} / 6$, and the corresponding expansions. The process needs to be monitored for a number of reasons. One has to make sure that no singularities occur during the integration process defining $J_{0}(u)$ : the Maple system elects not to make this assumption by itself, thus we compute $J_{0}(u)=\lim _{z \rightarrow 1} I_{1}(z ; u)$. Also, the normal forms used by the system for indefinite integration introduce the transformation $\log (1-u) \mapsto \log (u-1)+i \pi$; in order to recover the suitable branch of logarithms we perform a substitution that involves $\log (u-1) \mapsto \log (1-u)$ and $i \mapsto 0$, and whose validity can be checked via series expansions. Eventually, one arrives at the final form of $J_{0}(u)$,

$$
\begin{gathered}
{\left[\left(-2 u^{3}-4 u^{2}\right) \pi^{2}+\left(-24 u^{2}-6 u+30 u^{3}\right) \log (1-u)\right.} \\
\left.+\left(12 u^{3}+24 u^{2}\right) \operatorname{Li}_{2}(u)+4-15 u+54 u^{2}-43 u^{3}\right] / 12(1-u)^{4}
\end{gathered}
$$

The other two functions $J_{1}(u)$ and $J_{2}(u)$ surrender themselves similarly, and we derive a suitable form of $\gamma(u)$ after a couple of minutes of computing time and a couple of hours of human interaction. 